\section{Lower urinary cotinine level is associated with a trend toward more myopic refractive errors in Korean adolescents}

${ }^{1}$ Department of Family Medicine, Sahmyook Medical Center, Seoul, Republic of Korea

${ }^{2}$ Department of Ophthalmology and Visual Science, Seoul St. Mary's Hospital, College of Medicine, The Catholic University of Korea, Seoul, Republic of Korea

${ }^{3}$ Department of Ophthalmology and Visual Science, Uijeongbu St. Mary's Hospital, College of Medicine, The Catholic University of Korea, Seoul, Republic of Korea

${ }^{4}$ Department of Pediatrics, Incheon St. Mary's Hospital, College of Medicine, The Catholic University of Korea, Seoul, Republic of Korea

${ }^{5}$ Department of Biostatistics, The Catholic University of Korea, Seoul, Republic of Korea

Correspondence: SH Park, Department of Ophthalmology and Visual Science, Seoul St. Mary's Hospital, College of Medicine, The Catholic University of Korea, 222, Banpo-daero, Seocho-gu, Seoul 06591, Republic of Korea

Tel: +82-2-2258-6204;

Fax: +82-2-599-7405.

E-mail: vaccine@

catholic.ac.kr

Received: 28 October 2016 Accepted in revised form: 6 January 2017

Published online: 10 March 2017

\begin{abstract}
Purpose To investigate the association between urinary cotinine levels as an objective biological marker for exposure to nicotine and refractive status.

Patients and methods This cross-sectional study analyzed data from the Korea National Health and Nutrition Examination Survey between 2008 and 2011. A total of 1139 Korean adolescents aged 12-18 years were enrolled. Urinary cotinine concentrations and other potential risk factors for myopia were examined. Correlation analyses and multivariate regression analysis were performed to investigate the association between urinary cotinine level and refractive error.

Results Spherical equivalent correlated significantly with urinary cotinine concentration $(r=0.104, P=0.011)$. Lower urinary cotinine level was associated with a trend toward more myopic refractive errors ( $P$ for trend $=0.003$ ). After adjusting for age, sex, area of residence, physical activity, serum 25-hydroxyvitamin D level, parental income level, and receipt of basic livelihood security, subjects with a low urinary cotinine level had a significantly increased risk of myopia $<-0.5 \mathrm{D}$ (odds ratio (OR) 1.95, 95\% confidence interval (CI) 1.18-3.21), <-3.0 D (OR 2.03, 95\% CI 1.29-3.2), and $<-6.0 \mathrm{D}$ (OR $2.2,95 \% \mathrm{CI}, 1.15-4.23)$ when compared with subjects with a high urinary cotinine level. As urinary cotinine level decreased, the risks of myopia $<-0.5 \mathrm{D},<-3.0 \mathrm{D}$, and $<-6.0 \mathrm{D}$ increased significantly ( $P$ for trend $<0.05$ ). Conclusion A trend toward less myopic refractive error was observed among Korean adolescents with higher urinary cotinine levels. This result provides the epidemiologic evidence implying nicotine as a potential
\end{abstract}

GE Nam², BE Hwang², Y-C Lee³ , J-S Paik², S-W Yang ${ }^{2}$, Y-H Chun ${ }^{4}, \mathrm{~K} \mathrm{Han}^{5}$, YG Park ${ }^{5}$, SH Park ${ }^{2}$ and Epidemiologic Survey Committee of the Korean Ophthalmological Society

modulator related with refractive development. Further studies with full consideration for myopia-associated risk factors are required to yield clear answers on the direct effect of smoking to the refractive status.

Eye (2017) 31, 1060-1067; doi:10.1038/eye.2017.36; published online 10 March 2017

\section{Introduction}

Myopia is a complex disorder from both genetic and environmental causes. The prevalence of myopia varies by country and ethnic group. ${ }^{1-4}$ The incidence of myopia has been increasing in several Asian countries, including Korea; therefore, myopia has become an important public health concern. ${ }^{4-6}$ Recent worldwide increases in the prevalence of myopia strongly support a major role for environmental influences rather than genetic contributions in the regulation of ocular growth. ${ }^{7,8} \mathrm{~A}$ recent population-based study reported that the prevalence rates of myopia ( $<-0.5$ diopter (D)) and high myopia ( $<-6.0$ D) among 13-18-yearold Korean adolescents were $80.1 \%$ and $8.9 \%$, respectively. ${ }^{5}$ This increase in prevalence and severity of myopia as a major public health problem, should prompt political awareness of an 'epidemic' of myopia as a serious problem. ${ }^{9}$

The concept that the retina is central to the mechanism regulating emmetropization and underlying refractive errors has been supported. 9,10 The general roles of acetylcholine signaling through muscarinic and/or nicotinic acetylcholine receptors (nAChRs) and the retinal dopamine signaling pathway have been extensively studied relative to refractive development. ${ }^{9-14}$ Nicotine is a prominent constituent of tobacco smoke. nAChRs play a 
role from the earliest stages of development of the vertebrate retina to later stages of neuronal growth and synaptogenesis. ${ }^{15,16}$ Pharmacologic blockade of nAChRs affected the development of form-deprivation myopia with a dose-related response in chicks. ${ }^{13}$ Several epidemiological studies have shown an inverse relationship between parental smoking and childhood myopia in Caucasian, Egyptian and Singaporean populations. ${ }^{17-20}$ Although the underlying molecular basis to explain the effect of smoking on refractive development has not been clearly elucidated, the laboratory findings with consistent epidemiological associations imply that $\mathrm{nAchR}$ signaling would have a potential role to modulate the eye growth and subsequent refractive development.

Smoking status is most commonly self-reported through questionnaires and interviews because they are efficient and convenient to use and have relatively high accuracy. Passive smoking is defined as inhaling a mixture of smoke released by the burning end of a cigarette combined with cigarette smoke exhaled by the smoker. Nicotine can be quantified to correspond to the number of cigarettes smoked and nicotine absorbed by the body is primarily metabolized in the liver to cotinine. ${ }^{21,22}$ Cotinine is a major metabolite of nicotine and has been recognized as the most appropriate parameter to assess tobacco exposure and smoking status due to its high stability and long duration. ${ }^{21,22}$ Urinary cotinine has been used in many epidemiological surveys as an ideal biochemical marker for measuring the level of exposure to tobacco smoke..$^{23-25}$

The purpose of this study was to assess the association between urinary cotinine concentrations as an objective biological marker for exposure to nicotine and refractive errors among Korean adolescents based on data gathered from the Korea National Health and Nutrition Examination Survey (KNHANES) 2008-2011.

\section{Patients and methods}

\section{Data source and study participants}

The KNHANES is a nationwide, population-based, crosssectional health examination survey that has been conducted regularly since July 1998 by the Division of Chronic Disease Surveillance at the Korea Centers for Disease Control and Prevention and the Ministry of Health and Welfare to monitor the general health and nutritional status of the South Korean population. The KNHANES comprises a health interview survey, nutrition survey, and health examination survey. ${ }^{26,27}$ A stratified, multistage probability sampling design is used to select household units to participate in the survey. This survey has conducted ophthalmologic interviews and examinations with the participation of the Korean Ophthalmological Society since July $2008 .^{28}$

Data from the fourth (KNHANES IV-2 and 3, 2008 and 2009) and fifth (KNHANES V-1 and 2, 2010 and 2011) were used to estimate the association between urinary cotinine levels and refractive errors in Korean adolescents (mean study population age, $15.5 \pm 0.1$ years). Among the 1358 subjects aged $12-18$ years who had completed the health examination survey and underwent ophthalmological examinations and measurement of urinary cotinine, 219 were excluded for the following reasons: 27 subjects presented with ocular pathologies (strabismus or amblyopia), 103 subjects were current smokers based on their questionnaire responses, and 89 subjects had a urinary cotinine concentration compatible with active smoking (>50 ng/ml). ${ }^{22}$ The mean urinary cotinine concentration of 103 current smokers from selfreported questionnaire was $303 \mathrm{ng} / \mathrm{ml}$. Finally, 1139 adolescents aged 12-18 years were included in this study. Since all survey participants were considered minors, their parents signed written informed consent forms. The institutional review board of the Catholic University of Korea approved the informed consents and study protocol in accordance with the Declaration of Helsinki (IRB number: KC13EISI0636). The data are available from the Korean Centers for Disease Control and Prevention (http:/ / knhanes.cdc.go.kr).

\section{Demographic variables}

A detailed interviewer-administered questionnaire was administered to collect relevant demographic, socioeconomic, and medical information. Demographic variables, including age, sex, area of residence, parental income, receipt of national basic livelihood security, and physical activity, were obtained through direct interviews conducted according to standardized questionnaires. The participants were categorized into the low-income group if their parental incomes belonged to the lowest quartile. The national basic livelihood security means supplementary benefits to ensure that recipients reached the minimum income guaranteed by the government. Physical activity was estimated from self-reports using the short International Physical Activity Questionnaire modified for the Korean population. ${ }^{29}$ Moderate physical activity was defined as a positive answer regarding participation in moderate-intensity physical activity for $>20$ min per session more than three times per week. Moderate-intensity physical activity was defined as physical activity causing a slight increase in respiration or heart rate for at least $10 \mathrm{~min}$; examples include carrying light loads, cycling at a regular pace, or playing tennis. 


\section{Anthropometric and biochemical measurements}

Anthropometric measurements were made in a mobile screening bus 1 week before the interviewers visited the participants' homes. Height, body weight, and waist circumference (WC) were measured according to standard methods. Body mass index (BMI) was calculated as (weight in kilograms)/(height in meters). ${ }^{2}$ WC was measured to the nearest $0.1 \mathrm{~cm}$ at the narrowest point between the lowest rib and the uppermost lateral border of the right iliac crest at the end of a normal expiration.

Blood samples were obtained after fasting for at least 8 h. Serum 25-hydroxyvitamin D $(25[\mathrm{OH}] \mathrm{D})$ concentrations were measured by using gamma counter (1470 Wizard; PerkinElmer, Wallac, Turku, Finland) with a radioimmunoassay (RIA) using a 25(OH)D 125I RIA kit (DiaSorin, Stillwater, MN, USA).

Urine samples were collected from the subjects to measure urinary cotinine concentration. Gas chromatography and mass spectrometry was performed using a Clarus $600 \mathrm{~T}$ analyzer (PerkinElmer, Turku, Finland). The urinary cotinine detection limit was $0.26 \mathrm{ng} / \mathrm{ml}$ and mean recovery was $98.5 \%$. The coefficients of variation were $3.43-9.11 \%$ in 2008 , $0.79-8.17 \%$ in $2009,0.48-5.29 \%$ in 2010 , and $0.34-2.82 \%$ in 2011 based on conventional standard control reference materials (ClinChek, RECIPE, Munich, Germany) for internal quality assurance and control. Subjects were categorized into tertiles $(\mathrm{T})$ of urinary cotinine level with age-specific cut-off values.

\section{Ophthalmologic examinations}

The participants underwent full ophthalmological evaluations that included non-cycloplegic autorefraction, visual acuity testing, intraocular pressure measurements, slit-lamp examinations, and fundus photographs. An autorefractor-keratometer (KR 8800; Topcon, Tokyo, Japan) was used for all refraction measurements. Spherical equivalents (SE) were calculated as the spherical value plus half of the cylindrical value.

\section{Statistical analyses}

Statistical analyses were performed with the SAS survey procedure (version 9.2; SAS Institute, Cary, NC, USA) to incorporate sample weights and adjust the analysis of the complex KNHANES survey sample design. The procedures included unequal selection probabilities, oversampling, and non-responses so that inferences could be made regarding Korean adolescent participants. The statistical analysis was performed only for right eyes. The right and left eye SE correlation was high (Pearson's correlation coefficient $(r)=0.93)$.
The participants' characteristics are described as means and standard errors for continuous variables and as percentages and standard errors for categorical variables. We used the $\chi^{2}$ test to examine categorical variables and Student's $t$-test and analysis of variance to explore continuous variables. Correlation analyses were performed by calculating Pearson's correlation coefficients $(r)$. A scatter diagram was prepared to show the correlation between the degree of refractive error and urinary cotinine level for all adolescents.

Mean SE values according to urinary cotinine levels were assessed by analysis of covariance. We initially performed an unadjusted analysis in model 1 and then adjusted for age and sex in model 2. In model 3, additional adjustment was performed for potential confounding variables, such as area of residence, parental income, receipt of national basic livelihood security, physical activity, and serum vitamin D level. A multivariate logistic regression analysis was performed to estimate the odds ratios (OR) and 95\% confidential intervals $(\mathrm{CI})$ for the incidence of myopia $<-0.5 \mathrm{D}$, $<-3.0 \mathrm{D}$, and $<-6.0 \mathrm{D}$ in each model described above. A $P$-value $<0.05$ was considered significant.

\section{Results}

\section{Baseline characteristics of study subjects}

A total of 1139 eligible adolescents between 12 and 18 years of age were examined. Table 1 shows the basic characteristics of the study subjects according to urinary cotinine level. Mean refractive errors were $-3.1 \mathrm{D},-2.8 \mathrm{D}$, and $-2.2 \mathrm{D}$ in the low (T1), middle (T2), and high (T3) urinary cotinine level groups, respectively $(P=0.002)$. Sex, age, area of residence, parental income, receipt of national basic livelihood security, physical activity, serum $25(\mathrm{OH}) \mathrm{D}$ level, and the anthropometric measures, including height, weight, BMI, and WC were not different among the low, middle, and high urinary cotinine level groups.

\section{Association between urinary cotinine level and refractive errors}

The distributions of refractive errors in the SE according to urinary cotinine level are shown in Figure 1. The prevalence rates of mild $(-0.5$ to $-3.0 \mathrm{D})$, moderate $(-3.0$ to $-6.0 \mathrm{D})$, and high myopia $(<-6.0 \mathrm{D})$ were $35.1 \%$, $30.1 \%$, and $14.8 \%$, respectively, in subjects with low urinary cotinine level (T1). A trend toward decreased myopia was observed in subjects with higher urinary cotinine level ( $P$ for trend $=0.003$ ). Table 2 shows the mean refractive error values according to urinary cotinine 
Table 1 Baseline characteristics of subjects according to urinary cotinine level

\begin{tabular}{|c|c|c|c|c|}
\hline & \multicolumn{4}{|c|}{ Urinary cotinine level $(\mathrm{ng} / \mathrm{ml})$} \\
\hline & $\mathrm{T} 1$ & $\mathrm{~T} 2$ & T3 & P-value \\
\hline Unweighted $(n)$ & 378 & 382 & 379 & - \\
\hline Sex $(\text { male })^{\mathrm{a}}$ & $51.5(3.2)$ & $53.6(3.4)$ & $54.1(3.1)$ & $0.848^{\mathrm{b}}$ \\
\hline Age (years) ${ }^{c}$ & $14.8 \pm 0.1$ & $14.9 \pm 0.1$ & $14.9 \pm 0.1$ & $0.86^{\mathrm{d}}$ \\
\hline Area of residence (rural) ${ }^{\mathrm{a}}$ & $16.3(3.3)$ & $20.5(3.7)$ & $13.6(2.9)$ & $0.229^{\mathrm{b}}$ \\
\hline Physical activity (yes) ${ }^{a}$ & $27.3(3.4)$ & $29.9(3.2)$ & $32.5(3.7)$ & $0.596^{\mathrm{b}}$ \\
\hline $\begin{array}{l}\text { Parental income level } \\
\text { (the lowest quartile) }^{\mathrm{a}}\end{array}$ & $10.5(2.1)$ & $14.5(2.7)$ & $13.0(3.7)$ & $0.6^{\mathrm{b}}$ \\
\hline Receipt of national basic livelihood security (yes) ${ }^{\mathrm{a}}$ & $3.9(1.3)$ & $5.6(1.9)$ & $10.0(3.6)$ & $0.152^{\mathrm{b}}$ \\
\hline Height $(\mathrm{cm})^{\mathrm{c}}$ & $164.6 \pm 0.6$ & $163.5 \pm 0.6$ & $164.5 \pm 0.7$ & $0.363^{\mathrm{d}}$ \\
\hline Body weight $(\mathrm{kg})^{c}$ & $56.3 \pm 0.9$ & $57.1 \pm 0.8$ & $57.3 \pm 1.2$ & $0.748^{\mathrm{d}}$ \\
\hline BMI $\left(\mathrm{kg} / \mathrm{m}^{2}\right)^{\mathrm{c}}$ & $20.6 \pm 0.2$ & $21.2 \pm 0.2$ & $21.0 \pm 0.3$ & $0.221^{\mathrm{d}}$ \\
\hline $\mathrm{WC}(\mathrm{cm})^{\mathrm{c}}$ & $69.8 \pm 0.7$ & $70.7 \pm 0.7$ & $70.4 \pm 0.9$ & $0.664^{\mathrm{d}}$ \\
\hline SE (diopter) ${ }^{c}$ & $-3.1 \pm 0.2$ & $-2.8 \pm 0.1$ & $-2.2 \pm 0.1$ & $0.002^{\mathrm{d}}$ \\
\hline $25(\mathrm{OH}) \mathrm{D}(\mathrm{ng} / \mathrm{ml})^{\mathrm{c}}$ & $16.4 \pm 0.4$ & $16.3 \pm 0.3$ & $16.1 \pm 0.4$ & $0.875^{\mathrm{d}}$ \\
\hline
\end{tabular}

Abbreviations: 25(OH)D, 25-hydroxyvitamin D; ANOVA, analysis of variance; BMI, body mass index; SE, spherical equivalent; WC, waist circumference. ${ }^{a}$ Values are shown as $\%$ (standard errors). ${ }^{b} P$-values were calculated by using $\chi^{2}$ test. ${ }^{c}$ Values are shown as means \pm standard errors. ${ }^{d} P$-values were calculated by using analysis of variance test.

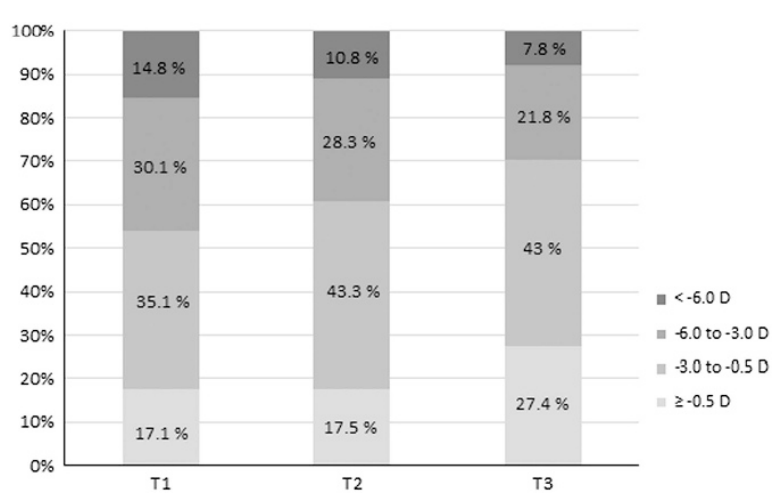

Figure 1 Distribution of refractive errors in spherical equivalents according to urinary cotinine level ( $\mathrm{D}=$ diopter, $P$ for trend $=0.003$ ).

level. The mean refractive error value was significantly less myopic in subjects with higher urinary cotinine level, regardless of adjustment for confounding variables $(P$ for trend $=0.001$ in model 1 and model 2, and $<0.001$ in model 3). Refractive error in the SE correlated significantly with urinary cotinine concentration (Figure 2; $r=0.104, P=0.011$ ). Low urinary cotinine level was associated with a trend toward more myopic refractive errors.

\section{Risk of myopia according to urinary cotinine level}

The ORs of myopia ( $<-0.5 \mathrm{D},<-3.0 \mathrm{D}$, and $<-6.0 \mathrm{D})$ in relation to urinary cotinine level are presented in Table 3. In unadjusted analysis (model 1), subjects with a low urinary cotinine level (T1) had an OR of 1.94 (95\% CI 1.17-3.2, $P$ for trend $=0.011)$ for myopia $<-0.5 \mathrm{D}, 1.91$
$(95 \%$ CI 1.22-2.99, $P$ for trend $=0.005)$ for myopia $<-3.0$ $\mathrm{D}$, and $1.91(95 \%$ CI 1.01-3.58, $P$ for trend $=0.046)$ for myopia $<-6.0 \mathrm{D}$, compared to those with high urinary cotinine levels (T3). After adjusting for age and sex (model 2), this association remained significant. Even after making additional adjustments for potential confounding variables such as area of residence, parental income, receipt of national basic livelihood security, physical activity, and serum vitamin D level (model 3), subjects with low urinary cotinine levels (T1) also had an increased risk of myopia: $<-0.5 \mathrm{D}$ (OR 1.95, 95\% CI 1.18-3.21), <-3.0 D (OR 2.03, 95\% CI 1.29-3.2), and $<-6.0 \mathrm{D}$ (OR 2.2, 95\% CI 1.15-4.23) in comparison to subjects with high urinary cotinine levels (T3). As urinary cotinine level decreased, the risk of myopia $<-0.5 \mathrm{D},<-3.0 \mathrm{D}$, and $<-6.0 \mathrm{D}$ tended to increase $(P$ for trend $=0.009,0.003$, and 0.038 , respectively).

\section{Discussion}

Our results suggest an inverse association between urinary cotinine level and myopic refractive errors in Korean adolescents. Subjects with a lower urinary cotinine level were more likely to be myopic. Urinary cotinine level was an independent factor associated with refractive error after adjustment for potential confounding factors, such as age, sex, area of residence, parental income, receipt of national basic livelihood security, physical activity, and serum vitamin D level.

Myopia is a multifactorial disease with both genetic and various environmental causes for development and progression. ${ }^{1,7}$ Reduced outdoor activity, excessive near 
Table 2 Mean values of refractive error (D) according to urinary cotinine level

\begin{tabular}{lcccc}
\hline & \multicolumn{4}{c}{ Urinary cotinine level $(\mathrm{ng} / \mathrm{ml})$} \\
\cline { 2 - 5 } & $T 1$ & $T 2$ & $T 3$ & P for trend $^{\mathrm{a}}$ \\
\hline Model 1 $^{\mathrm{b}}$ & $-3.10 \pm 0.22$ & $-2.7 \pm 0.19$ & $-2.16 \pm 0.17$ & 0.001 \\
Model 2 $^{\mathrm{c}}$ & $-3.06 \pm 0.21$ & $-2.64 \pm 0.18$ & $-2.09 \pm 0.18$ & 0.001 \\
Model 3 $^{\mathrm{d}}$ & $-3.08 \pm 0.21$ & $-2.87 \pm 0.18$ & $-2.04 \pm 0.18$ & $<0.001$ \\
\hline
\end{tabular}

Abbreviation: D, diopter. ${ }^{a} P$-values were calculated by using analysis of covariance (ANCOVA) test and $P$ for trend was obtained from general linear model. ${ }^{b}$ Model 1 was unadjusted. ${ }^{c}$ Model 2 was adjusted for age and sex. ${ }^{\mathrm{d}}$ Model 3 was adjusted for age, sex, area of residence, parental income level, receipt of basic livelihood security, physical activity, and serum 25-hydroxyvitamin D level. Values are shown as least-squared means \pm standard errors.

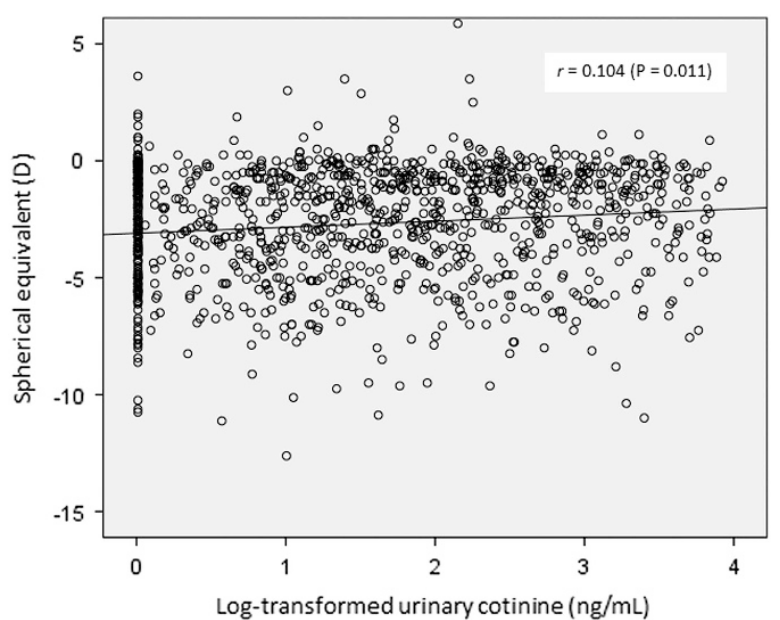

Figure 2 Scatter plot of refractive errors and urinary cotinine levels in Korean adolescents.

work, and a higher education level are major factors associated with myopia. ${ }^{1,7,8}$ Cigarette smoking has not been a major target of interest with regard to refractive development. Several studies have investigated the relationship between parental smoking and childhood myopia. ${ }^{17-20}$ The first epidemiologic study on this relationship in Singaporean Chinese children aged 7-9 years suggested an association of more hyperopic refractions and maternal smoking, but not paternal smoking. ${ }^{17}$ A population-based study in young Singaporean Chinese children aged 6-72 months revealed an inverse relationship between parental (maternal and paternal) smoking and incidence of myopia. ${ }^{18}$ Another study including mainly Caucasians and AfricanAmericans from the United States also demonstrated the association of less myopia and more hyperopia refraction with prenatal and childhood exposure to tobacco smoke. ${ }^{19}$ These three epidemiological studies were based on questionnaires to obtain information on parental smoking history. A recent Egyptian study using urinary cotinine and the cotinine/creatinine ratio as chemical parameters to assess smoke exposure also found that
Table 3 ORs (95\% CIs) of myopia in relation to urinary cotinine level in Korean adolescents

\begin{tabular}{lccc}
\hline \multicolumn{5}{c}{ Myopia $<-0.5 \mathrm{D}$} & Myopia $<-3.0 \mathrm{D}$ & Myopia $<-6.0 \mathrm{D}$ \\
\hline Model $1^{\mathrm{a}}$ & & & \\
$\mathrm{T} 1$ & $1.94(1.17-3.2)$ & $1.91(1.22-2.99)$ & $1.91(1.01-3.58)$ \\
$\mathrm{T} 2$ & $1.67(0.99-2.84)$ & $1.57(1.05-2.36)$ & $1.29(0.67-2.48)$ \\
$\mathrm{T} 3$ & 1 & 1 & 1 \\
$P$ for trend & 0.011 & 0.005 & 0.046 \\
& & & \\
Model 2 & & & \\
T1 & $1.93(1.18-3.17)$ & $1.94(1.24-3.04)$ & $2(1.04-3.82)$ \\
T2 & $1.68(0.99-2.85)$ & $1.59(1.05-2.39)$ & $1.33(0.68-2.59)$ \\
T3 & 1 & 1 & 1 \\
$P$ for trend & 0.011 & 0.004 & 0.037 \\
& & & \\
Model 3 & & & \\
T1 & $1.95(1.18-3.21)$ & $2.03(1.29-3.2)$ & $2.2(1.15-4.23)$ \\
T2 & $1.8(1.09-2.98)$ & $1.71(1.14-2.57)$ & $1.34(0.67-2.67)$ \\
T3 & 1 & 1 & 1 \\
$P$ for trend & 0.009 & 0.003 & 0.038 \\
\hline
\end{tabular}

Abbreviation: D, diopter. ${ }^{\mathrm{a} M o d e l} 1$ was unadjusted. ${ }^{\mathrm{b}}$ Model 2 was adjusted for age and sex. 'Model 3 was adjusted for age, sex, area of residence, parental income level, receipt of basic livelihood security, physical activity, and serum 25-hydroxyvitamin D level.

exposing children to passive smoking might be associated with a refractive error shift toward hypermetropia. ${ }^{20}$ Our study is the first large scale population-based study to investigate the association between urinary cotinine level and refractive error in adolescents using nationally representative data. Urinary cotinine concentration was used in our study as a biomarker for exposure to nicotine. Urinary cotinine has been used in many epidemiological surveys as the ideal biochemical marker for measuring the level of exposure to tobacco smoke. ${ }^{23-25}$

Although inverse relationship between urinary cotinine level and myopic status was observed in the present study, it should be considered whether it was resulted from a direct effect of nicotine on refractive development or from a confounding association between parental socioeconomic status (SES) and smoking. The greater 
prevalence of myopia in population with higher SES factors was observed in a previous Korean study using KNHANES data. ${ }^{30}$ A strong negative association between parental SES and smoking has been reported in the previous studies. ${ }^{31-33}$ A recent Korean study using data from another survey also confirmed that lower SES was associated with higher odds of household secondhand smoke exposure in Korean adolescents. ${ }^{34}$ Increased parental enthusiasm for children's education in high SES populations could affect the myopic development.

Therefore, confounding effects of parental SES on passive smoking may underlie in the inverse association between urinary cotinine concentration and myopia. In the present study using KNHANES data, parental income level and status of receipt of basic livelihood security were selected to adjust parental SES. After adjustment for these potential confounding factors (model 3), subjects with low urinary cotinine levels (T1) also had an increased risk of myopia.

Recent systematic review and meta-analysis reported that fetal exposure to cigarette smoking is risk factor for childhood visual problems particularly in hyperopia, amblyopia, and strabismus. ${ }^{35,36}$ Children whose mother smoked during pregnancy were 1.43 times more likely to suffer from hyperopia compared with children whose mother did not smoke. ${ }^{35}$ Intrauterine exposure to maternal smoking may have possibly protecting effects for later development of myopia.

Our findings are consistent with results from previous epidemiological studies and support that nAChRs might play a role in human refractive development. $\mathrm{nAChRs}$ are ligand-gated cation channels consisting of pentameric complexes. nAChRs play a role from the earliest stages of development of the vertebrate retina to later stages of neuronal growth and synaptogenesis. ${ }^{15,16}$ Nicotine stimulates retinal angiogenesis, accelerates diabetic retinal changes, alters retinal pigment epithelial cell morphology and function, and modifies electroretinogram responses. ${ }^{37-42}$ The expression of different $\mathrm{nAChR}$ subtypes in retinal stem cells and retinal progenitors is specifically distributed and is likely to be differentially regulated throughout retinal development. ${ }^{43,44}$ Visual experience affects the expression of retinal $\mathrm{nAchRs}$ during postnatal retinal development in a rat model. ${ }^{45}$ The recent confirmation of expression of diverse $\mathrm{nAChR}$ subunits in different retinal neuron classes in the Rhesus monkey retina suggests that nicotinic cholinergic signaling in the retina may influence not only visual processing and but also normal ocular growth and resulting refractive development. ${ }^{41}$ Nicotinic antagonists affect eye growth, influencing the development of form-deprivation myopia and normal eye growth in young chicks with multiphasic dose-response curves. ${ }^{13}$ Future experimental researches are required to reveal whether and how nicotine affects ocular growth and refractive development.

Our study had some potential limitations. The KNHANES was not planned specifically for an ophthalmological evaluation of adolescents. The measurement of refraction was performed without cycloplegia, which led to some over-estimation of the prevalence of myopia in children and younger adults, to the erroneous classification of hyperopic and emmetropic subjects, and to marked errors in the estimation of mean $\mathrm{SE}$ in a sample. Although these limitations may have affected the final results, the direction of the association between urinary cotinine level and refractive errors was reliable. Moreover, KNHANES has not collected data on the major risk factors for myopia, such as outdoor activity, near work, academic performance, and educational attainment phenotype of subjects at school and parental myopia and educational level. As outdoor activity or sun exposure duration of adolescent age group was lacked in data of KNHANES, we considered an adjustment of physical activity and serum 25(OH)D level instead of them. Another limitation of this study was the cross-sectional design, as causal relationships cannot be established. As this was an epidemiological study, the underlying mechanism concerning the association between passive smoking and the development and progression of myopia was unknown. Nonetheless, the great strength of this study is that it is the first study to assess the association between urinary cotinine level as a chemical biomarker for exposure to nicotine and refractive errors among nationally representative data with a relatively large sample size. Further studies using cycloplegic refraction with consideration for myopia-associated risk factors for the child and adolescent participants are required to yield clear answers on the effect of smoking to the refractive status.

In conclusion, a trend toward less myopic refractive error was observed among Korean adolescents with higher urinary cotinine levels. This population-based study extends the previously observed association between passive smoking and refractive error in adolescent age group, and implies nicotine as a potential modulator related with refractive development. However, this inverse relationship should be interpreted with considering that it could still be confounded as many of confounding factors which were not measured in this study. Further studies are warranted to better understand the role of $\mathrm{nAChRs}$ in refractive development that may help to establish the targeted strategies for prevention of myopia. 


\section{Summary}

\section{What was known before}

- Several epidemiological studies have suggested an inverse relationship between parental smoking and childhood myopia.

\section{What this study adds}

- This study is the first large scale population-based study to show the inverse association between urinary cotinine level as a biomarker for exposure to nicotine and refractive error in adolescents using nationally representative data.

- Lower urinary cotinine level was associated with a trend toward more myopic refractive errors.

- This result provides the epidemiologic evidence implying that nicotinic cholinergic signaling would have a potential role to modulate the eye growth and subsequent refractive development.

\section{Conflict of interest}

The authors declare no conflict of interest.

\section{Acknowledgements}

The authors appreciate the staffs and participants in the KNHANES 2008-2011.

\section{References}

1 Pan CW, Ramamurthy D, Saw SM. Worldwide prevalence and risk factors for myopia. Ophthalmic Physiol Opt 2012; 32 3-16.

2 Vitale S, Sperduto RD, Ferris FL 3rd. Increased prevalence of myopia in the United States between 1971-1972 and 1999-2004. Arch Ophthalmol 2009; 127: 1632-1639.

3 Sawada A, Tomidokoro A, Araie M, Iwase A, Yamamoto T. Refractive errors in an elderly Japanese population: the Tajimi study. Ophthalmology 2008; 115: 363-370, e3.

4 Pan CW, Zheng YF, Anuar AR, Chew M, Gazzard G, Aung $\mathrm{T}$ et al. Prevalence of refractive errors in a multiethnic Asian population: the Singapore epidemiology of eye disease study. Invest Ophthalmol Vis Sci 2013; 54: 2590-2598.

5 Choi JA, Han K, Park YM, La TY. Low serum 25hydroxyvitamin D is associated with myopia in Korean adolescents. Invest Ophthalmol Vis Sci 2014; 55: 2041-2047.

6 Kim EC, Morgan IG, Kakizaki H, Kang S, Jee D. Prevalence and risk factors for refractive errors: Korean National Health and Nutrition Examination Survey 2008-2011. PLoS One 2013; 8: e80361.

7 Saw SM. A synopsis of the prevalence rates and environmental risk factors for myopia. Clin Exp Optom 2003 86: 289-294.

8 Dirani M, Tong L, Gazzard G, Zhang X, Chia A, Young TL et al. Outdoor activity and myopia in Singapore teenage children. Br J Ophthalmol 2009; 93: 997-1000.

9 Stone RA, Pardue MT, Iuvone PM, Khurana TS. Pharmacology of myopia and potential role for intrinsic retinal circadian rhythms. Exp Eye Res 2013; 114: 35-47.

10 Feldkaemper M, Schaeffel F. An updated view on the role of dopamine in myopia. Exp Eye Res 2013; 114: 106-119.
11 Duncan G, Collison DJ. Role of the non-neuronal cholinergic system in the eye: a review. Life Sci 2003; 72: 2013-2019.

12 Arumugam B, McBrien NA. Muscarinic antagonist control of myopia: evidence for M4 and M1 receptor-based pathways in the inhibition of experimentally-induced axial myopia in the tree shrew. Invest Ophthalmol Vis Sci 2012; 53: 5827-5837.

13 Stone RA, Sugimoto R, Gill AS, Liu J, Capehart C, Lindstrom JM. Effects of nicotinic antagonists on ocular growth and experimental myopia. Invest Ophthalmol Vis Sci 2001; 42: 557-565.

14 McBrien NA, Jobling AI, Truong HT, Cottriall CL, Gentle A. Expression of muscarinic receptor subtypes in tree shrew ocular tissues and their regulation during the development of myopia. Mol Vis 2009; 15: 464-475.

15 Zhou ZJ. The function of the cholinergic system in the developing mammalian retina. Prog Brain Res 2001; 131: 599-613.

16 Feller MB. The role of nAChR-mediated spontaneous retinal activity in visual system development. J Neurobiol 2002; 53: 556-567.

17 Saw SM, Chia KS, Lindstrom JM, Tan DT, Stone RA. Childhood myopia and parental smoking. Br J Ophthalmol 2004; 88: 934-937.

18 Iyer JV, Low WC, Dirani M, Saw SM. Parental smoking and childhood refractive error: the STARS study. Eye (Lond) 2012; 26: 1324-1328.

19 Stone RA, Wilson LB, Ying GS, Liu C, Criss JS, Orlow J et al. Associations between childhood refraction and parental smoking. Invest Ophthalmol Vis Sci 2006; 47: 4277-4287.

20 El-Shazly AA. Passive smoking exposure might be associated with hypermetropia. Ophthalmic Physiol Opt 2012; 32: 304-307.

21 Benowitz NL, Jacob P 3rd. Metabolism of nicotine to cotinine studied by a dual stable isotope method. Clin Pharmacol Ther 1994; 56: 483-493.

22 SRNT Subcommittee on Biochemical Verification. Biochemical verification of tobacco use and cessation. Nicotine Tob Res 2002; 4: 149-159.

23 Haufroid V, Lison D. Urinary cotinine as a tobacco-smoke exposure index: a minireview. Int Arch Occup Environ Health 1998; 71: 162-168.

24 Park MB, Nam EW, Lee SK, Kim CB, Ranabhat C. The correlation of different cotinine levels with questionnaire results: a comparative study for different measurement methods of the adolescent smoking rate in Korea. Asia Pac J Public Health 2015; 27: 542-550.

25 Leone A. Biochemical markers of cardiovascular damage from tobacco smoke. Curr Pharm Des 2005; 11: 2199-2208.

26 Kim Y. The Korea National Health and Nutrition Examination Survey (KNHANES): current status and challenges. Epidemiol Health 2014; 36: e2014002.

27 Kweon S, Kim Y, Jang MJ, Kim Y, Kim K, Choi S et al. Data resource profile: the Korea National Health and Nutrition Examination Survey (KNHANES). Int J Epidemiol 2014; 43: 69-77.

28 Yoon KC, Mun GH, Kim SD, Kim SH, Kim CY, Park KH et al. Prevalence of eye diseases in South Korea: data from the Korea National Health and Nutrition Examination Survey 2008-2009. Korean J Ophthalmol 2011; 25: 421-433.

29 Oh JY, Yang YJ, Kim BS, Kang JH. Validity and reliability of korean version of international physical activity questionnaire (IPAQ) short form. J Korean Acad Fam Med 2007; 28: 532-541. 
30 Lim HT, Yoon JS, Hwang SS, Lee SY. Prevalence and associated sociodemographic factors of myopia in Korean children: the 2005 third Korea National Health and Nutrition Examination Survey (KNHANES III). Jpn J Ophthalmol 2012; 56: 76-81.

31 Laaksonen M, Rahkonen O, Karvonen S, Lahelma E. Socioeconomic status and smoking: analysing inequalities with multiple indicators. Eur J Public Health 2005; 15: 262-269.

32 Siahpush M, Borland R, Scollo M. Smoking and financial stress. Tob Control 2003; 12: 60-66.

33 Cavelaars AE, Kunst AE, Geurts JJ, Crialesi R, Grotvedt L, Helmert $U$ et al. Educational differences in smoking: international comparison. BMJ 2000; 320: 1102-1107.

34 Park S, Lim S, Kim J, Lee H, June KJ. Socioeconomic disparities in household secondhand smoke exposure among non-smoking adolescents in the Republic of Korea. Glob Public Health 2015; e-pub ahead of print 13 December 2015; doi: 10.1080/17441692.2015.1117119.

35 Li L, Qi Y, Shi W, Wang Y, Liu W, Hu M. A meta-analysis for assciation of maternal smoking with childhood refractive error and amblyopia. J Ophthalmol 2016; 2016: 8263832.

36 Fernandes M, Yang X, Li JY, Cheikh Ismail L. Smoking during pregnancy and vision difficulties in children: a systematic review. Acta Ophthalmol 2015; 93: 213-223.

37 Lecchi M, McIntosh JM, Bertrand S, Safran AB, Bertrand D. Functional properties of neuronal nicotinic acetylcholine receptors in the chick retina during development. Eur J Neurosci 2005; 21: 3182-3188.
38 Heeschen C, Jang JJ, Weis M, Pathak A, Kaji S, Hu RS et al. Nicotine stimulates angiogenesis and promotes tumor growth and atherosclerosis. Nat Med 2001; 7: 833-839.

39 Suner IJ, Espinosa-Heidmann DG, Marin-Castano ME, Hernandez EP, Pereira-Simon S, Cousins SW. Nicotine increases size and severity of experimental choroidal neovascularization. Invest Ophthalmol Vis Sci 2004; 45: 311-317.

40 Yang L, Gong H, Wang Y, Wang Y, Yin H, Chen P et al. Nicotine alters morphology and function of retinal pigment epithelial cells in mice. Toxicol Pathol 2010; 38: 560-567.

41 Varghese SB, Reid JC, Hartmann EE, Keyser KT. The effects of nicotine on the human electroretinogram. Invest Ophthalmol Vis Sci 2011; 52: 9445-9451.

42 Boretsky A, Gupta P, Tirgan N, Liu R, Godley BF, Zhang W et al. Nicotine accelerates diabetes-induced retinal changes. Curr Eye Res 2015; 40: 368-377.

43 Das AV, Edakkot S, Thoreson WB, James J, Bhattacharya S, Ahmad I. Membrane properties of retinal stem cells/ progenitors. Prog Retin Eye Res 2005; 24: 663-681.

44 Moretti M, Vailati S, Zoli M, Lippi G, Riganti L, Longhi R et al. Nicotinic acetylcholine receptor subtypes expression during rat retina development and their regulation by visual experience. Mol Pharmacol 2004; 66: 85-96.

45 Liu J, McGlinn AM, Fernandes A, Milam AH, Strang CE, Andison ME et al. Nicotinic acetylcholine receptor subunits in rhesus monkey retina. Invest Ophthalmol Vis Sci 2009; 50: 1408-1415. 